\title{
Representation of Curved Surfaces in Responses of Mechanoreceptive Afferent Fibers Innervating the Monkey's Fingerpad
}

\author{
A. W. Goodwin, A. S. Browning, and H. E. Wheat \\ Department of Anatomy and Cell Biology, University of Melbourne, Parkville, Victoria 3052, Australia
}

The aim was to elucidate how the population of digital nerve afferents signals information about the shape of objects in contact with the fingerpads during fine manipulations. Responses were recorded from single mechanoreceptive afferent fibers in median nerves of anesthetized monkeys. Seven spherical surfaces were used, varying from a highly curved surface (radius, $1.44 \mathrm{~mm}$; curvature, $694 \mathrm{~m}^{-1}$ ) to a flat surface (radius, $\infty$; curvature, $0 \mathrm{~m}^{-1}$ ). These were applied to the fibers' receptive fields, which were located on the central portion of a fingerpad. When the objects were located at the centers of the receptive fields, the responses of the slowly adapting fibers (SAls) increased as the curvature of the surface increased and as the contact force increased. All SAls behaved in the same way, differing only by a scaling factor (the sensitivity of the individual afferent). Responses of the rapidly adapting afferents were small and did not vary systematically with the stimulus parameters, and most Pacinians did not respond at all. Stimuli were applied at different positions in the receptive fields of SAls to define the response profiles of the afferents (response as a function of position on the fingerpad). All SAls had similarly shaped profiles for the same surface curvature and the shape differed for different curvatures. These profiles reflected the shape of the stimulus. An increase in contact force scaled these profiles upward. Thus, the population of digital nerve fibers signals unambiguous information about the shape and contact force of curved surfaces contacting the fingerpad.

[Key words: cutaneous mechanoreceptors, shape, monkey fingerpad, curvature, contact force, digital nerve]

Humans have the capacity to manipulate objects using skilled independent movements of the fingers and the apposable thumb. For such precise movements, the sensorimotor control system must have information about the local shape of the object, the contact force, and the position of the object on the fingers. While it has been recognized for a long time that joint receptors, muscle spindle afferents, and Golgi tendon organs contribute important signals during such tasks, the role of cutancous input has only been emphasized more recently (Burgess et al., 1982; Edin and Abbs, 1991). Most psychophysical studies of tactile shape perception have used active touch, which results in input from all

Received May 11, 1994; revised July 1, 1994; accepted July 14, 1994

This research was supported by a grant from the National Health and Medical Research Council of Australia.

Correspondence should be addressed to A. W. Goodwin at the above address. Copyright (C) 1995 Society for Neuroscience $0270-6474 / 95 / 150798-13 \$ 05.00 / 0$ the above receptors (Lederman and Klatzky, 1987; Roland and Mortensen, 1987). A recent study used passive touch in which only the cutaneous receptors in the fingerpads could convey information about the stimuli; these experiments showed that information about the local shape of contacted objects is accurately signaled (LaMotte and Srinivasan, 1987a; Srinivasan and LaMotte, 1987). The first clear indication, from neural recordings, that shape affects cutaneous mechanoreceptor responses was the observation of edge enhancement in slowly adapting type I afferents (Vierck, 1979; Phillips and Johnson, 1981 a; Johansson et al., 1982). In a more direct approach LaMotte and Srinivasan (LaMotte and Srinivasan, 1987a,b, 1993; Srinivasan and LaMotte, 1987) used cylinders and sinusoidally shaped steps stroked across the skin and indented into the skin to show the importance of the shape of the stimulus on the responses of cutaneous mechanoreceptive afferents. They showed that the curvature of the stimulus and its rate of change were prime determinants of responses.

There are many ways of quantifying three-dimensional shapes (Marshall, 1989). A parameter that is common to many of these definitions is the local curvature of the surface, and many algorithms proposed for extracting the shape of objects, either by the brain or by computational devices, are based on curvatures (Richards et al., 1986; DeVries et al., 1993). To facilitate analysis of the role of curvature, we have chosen to use spheres as our stimuli; these are objects of constant curvature. In previous psychophysical studies we have used spherical surfaces to measure the human capacity to perceive shape and contact force using the cutaneous receptors alone (Goodwin et al., 1991; Goodwin and Wheat, 1992a,b). The human performance was remarkable and it is not evident how the information was relayed by the peripheral nerves. In this study we have applied those same stimuli to the fingerpads of anesthetized monkeys to elucidate how such precise information about the shape and contact force is conveyed to the brain by the responses of the population of digital nerve fibers.

\section{Materials and Methods}

The methods for recording from single fibers in the peripheral nerve are standard and have been described previously (Goodwin and Morley, 1987); only a brief description is given here. Fourteen monkeys, 10 Macaca nemestrina and four Macaca fascicularis, with weights ranging from 3.3 to $6.7 \mathrm{~kg}$, were used. They were lightly anaesthetized with ketamine $(15 \mathrm{mg} / \mathrm{kg}, \mathrm{i} . \mathrm{m}$.) and given atropine $(60 \mu \mathrm{g} / \mathrm{kg}$, i.m.). Surgical anesthesia was induced by sodium pentobarbitone $(15-20 \mathrm{mg} / \mathrm{kg}, \mathrm{i} . \mathrm{v}$.) and an endotracheal cannula and intraperitoneal catheter were inserted. The catheter was used to maintain anesthesia throughout the experiment by regular doses of sodium pentobarbitone (approximately hourly) and for fluid replacement. Rectal temperature was monitored and was main- 
tained at $37^{\circ} \mathrm{C}$ by a heating blanket. Respiration rate, heart rate, blood pressure, end-tidal carbon dioxide levels, and oxygen saturation levels were also monitored. The experiment lasted up to $18 \mathrm{hr}$ and antibiotic cover was provided by amoxycillin $(18 \mathrm{mg} / \mathrm{kg}, \mathrm{i} . \mathrm{m}$.) every $6 \mathrm{hr}$ and, at the end of the experiment, by an intramuscular dose of $50 \mathrm{mg} / \mathrm{kg}$ benzathine penicillin plus $30 \mathrm{mg} / \mathrm{kg}$ procaine penicillin plus $19 \mathrm{mg} / \mathrm{kg}$ benzylpenicillin. Single fibers were isolated by exposure and microdissection of a median nerve first in the upper arm and then, after a recovery period of at least 2 weeks, in the lower arm. After at least 2 weeks the sequence was repeated for the other median nerve.

Single fibers were isolated, and slowly adapting type I afferents (SAIs) rapidly adapting afferents arising from Meissner corpuscles (RAs), and Pacinian afferents (PCs) were classified using the well-established criteria of responses to static stimuli, thresholds to rapidly changing or vibrating stimuli, and sizes of receptive fields (Talbot et al., 1968; Vallbo and Johansson, 1984). SAIs and RAs were accepted for study only if their receptive fields were located on the central region of a fingerpad. The PCs, as is typical of these afferents, had diffuse receptive fields; the most sensitive point in each field was estimated and if it was located on the glabrous skin of any phalanx of the finger then the PC was used. Data were gathered from 33 SAIs, 9 RAs, and 10 PCs.

The stimulator, which has been described previously (Goodwin et al., 1991), contained a balanced beam with attached objects that could be lowered to contact an immobilized finger. Contact force was controlled by changing the counterbalancing weight on the beam. The objects were locatcd on an indexed rotating hub so that they could be changed rapidly. The stimulator was mounted on an $\mathrm{x}-\mathrm{y}$ stage that could be positioned, with a resolution of $0.01 \mathrm{~mm}$, using micrometers and dial indicators The center of the receptive field of the isolated afferent was estimated as accurately as possible with a set of calibrated von Frey hairs. Then, the finger was embedded in modeling clay (plasticene) and the nail secured by cotton thread. The angle of the finger was adjusted so that the plane tangential to the fingerpad at the receptive field center was parallel to the experimental table, ensuring that the stimulator applied the objects normal to the skin at this point (Fig. 1). The hand was mounted on a micrometer monitored by a dial indicator (resolution, $0.01 \mathrm{~mm}$ ), allowing the finger to be raised until it just contacted the object, as judged visually through a magnifier. The hand was then lowered $0.5 \mathrm{~mm}$ and, when the stimulator was activated, the object moved through $0.5 \mathrm{~mm}$ to contact the skin. All the objects on the hub were set so that when positioned above the fingerpad, they were all the same distance $(0.5 \mathrm{~mm})$ from the skin and all contacted the receptive field center at the same point (see Goodwin et al., 1991, for details).

In the first series, seven different objects made from Delrin were used. The surfaces contacting the finger were all spherical with radii of 1.44 , $1.92,2.94,3.90,5.80,12.4 \mathrm{~mm}$ and $\infty$ (flat surface). The corresponding curvatures, given by the reciprocal of the radius, are $694,521,340,256$, $172,80.6$, and $0.00 \mathrm{~m}^{-1}$, respectively. They were applied to the fingerpad, at the estimated center of the receptive field, with contact forces of 10 or $20 \mathrm{gm}$ wt $(0.098$ or $0.196 \mathrm{~N}$, respectively). For any stimulus, the time sequence of presentation was as follows. $\dot{A}$ relay was energized on the stimulator, allowing the beam to move and apply the surface to the skin. After $4 \mathrm{sec}$ the relay was released, signaling the experimenter to raisc the stimulus off the skin and to reengage the beam. After a further $4 \mathrm{sec}$ the procedure was repeated twice. Thus, in each presentation there were three trials with $4 \mathrm{sec}$ of contact followed by $4 \mathrm{sec}$ of no contact. Eight blocks of data were collected; in each block each of the 14 curvature-force combinations occurred once with the order randomized. At the end of each block the height of the stimulus above the skin was checked and, if necessary, reset to $0.5 \mathrm{~mm}$. Thus, each stimulus was presented 24 times $(3 \times 8)$. This series took about $1.5 \mathrm{hr}$.

In the second series, the same seven surfaces as above were used but the contact force was $15 \mathrm{gm}$ wt $(0.147 \mathrm{~N})$. The stimuli were positioned in the receptive field at points on a $0.5 \mathrm{~mm}$ grid and the aim was to record from as many points as possible. Positions were defined by an $\mathrm{x}, \mathrm{y}$-coordinate system with the origin at the center of the receptive field and the $y$-axis parallel to the long axis of the finger (Fig. 1). The points were selected in the following order: points along the $y$-axis, then points along the $\mathrm{x}$-axis, and then as many points as possible along lines parallel to the $y$-axis at increasing distances from the origin. There were three factors that limited the maximum distance of the stimulus from the origin; their relevance varied with the location on the fingerpad of the center of the receptive field. First, on the proximal side, the surfaces could not be too close to the crease between the distal and middle phalanxes. Second, on the distal and two lateral sides, the curvature of
A

B
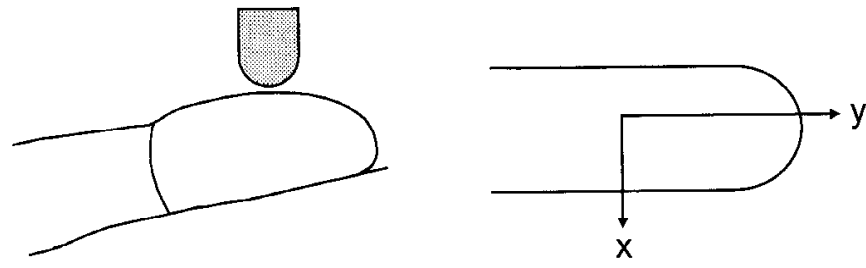

Figure 1. A, Objects with spherically curved surfaces were applied to the fingerpad. The curvature, contact force, and position on the finger could be varied. $B$, For each fiber, the position of the object on the fingerpad was defined by a rectangular coordinate system; the $x-$ and $y$-axes passed through the center of the receptive field with the $y$-axis parallel to the long axis of the finger and pointing distally.

the finger limited the distance. Third, the recording time from the fiber was limited-the largest field we mapped, with 66 points, required 9 hr. Because of time constraints, only one block of data was collected at each point. The block consisted of all seven surfaces presented in a random order. The time sequence was as above, namely, three trials, each with $4 \mathrm{sec}$ of contact followed by $4 \mathrm{sec}$ of no contact. $\Lambda t$ each point the height of the surface above the skin was set to $0.5 \mathrm{~mm}$. As a check on long-term stability of the afferent's responses, the block at the center of the receptive field was repeated over the data collection period at least three times.

In the third series, the two surfaces with radii of 1.92 and $5.80 \mathrm{~mm}$ (curvature, 521 and $172 \mathrm{~m}^{-1}$, respectively) were used with contact forces of $10,15,20$, and $25 \mathrm{gm}$ wt $(0.098,0.147,0.196$, and $0.245 \mathrm{~N}$, respectively). They were applied at points successively separated by 1.0 $\mathrm{mm}$ along the $\mathrm{y}$-axis. At each point, first the less curved surface was used at increasing contact force and then the more curved surface at increasing contact force, each combination being presented for three trials in the same time sequence as above. When all the chosen points along the $y$-axis had been completed, the whole sequence was repeated seven times.

The times of occurrence of action potentials and of the relay energizing pulse were recorded on a computer with a resolution of $0.1 \mathrm{msec}$.

\section{Results}

The beam of the stimulator was attached to a rotary damper. When the relay was energized, the stimulus surface moved through the gap of $0.5 \mathrm{~mm}$ to contact the skin at a constant velocity of about $20 \mathrm{~mm} / \mathrm{sec}$, coming to rest with a contact force equivalent to the weight placed on the beam. Typical responses for an SAI are shown in Figure 2, $A$ and $C$. As with other stimuli used by previous experimenters, there was an initial transient response followed by a static response for the duration of contact. Because of the damped nature of indentation, the dynamic component was not as marked as responses seen with step indentations of punctate probes (Cohen and Vierck, 1993a). The response waveform differed slightly from fiber to fiber and with different stimulus conditions. Two of the nine RAs studied did not respond at all to our stimuli even though they were sensitive to, and responded vigorously to, dynamically changing stimuli like light brush strokes or vibration. The responses in Figure 2, $B$ and $D$, are typical of the remaining seven RAs. Only a few action potentials were elicited during the initial phase of indentation.

As seen in Figure 2, there is a delay between the signal to the relay and the first action potential. Some of this is due to the latency of the receptor mechanisms and some to the time taken for the surface to travel the $0.5 \mathrm{~mm}$. To quantify the responses of the affcrents, wc computcd the total number of action potentials elicited in the first second commencing from the first 
A

B

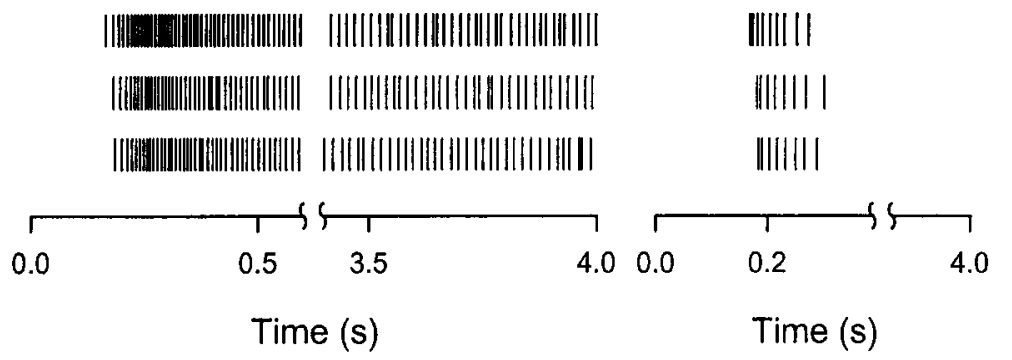

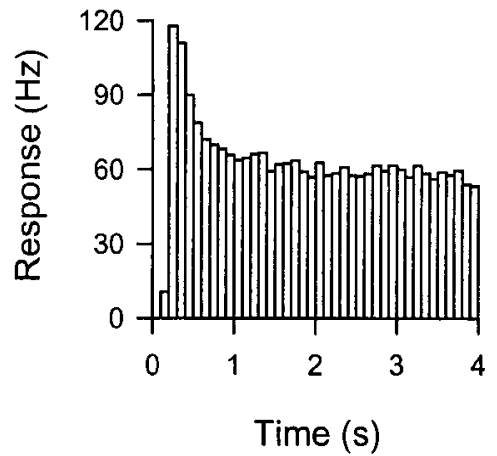

Figure 2. Typical responses of an SAI $(A$ and $C)$ and an RA $(B$ and $D)$ to our stimuli. $A$ and $B$, Vertical tick marks show the time of occurrence of action potentials in three successive trials. $C$ and $D$, Poststimulus histograms of response rate averaged over the three trials; bin width is 0.1 sec. The beam of the stimulator was released at time $t=0 \mathrm{sec}$. The curvature was $340 \mathrm{~m}^{-1}$ and the contact force was $20 \mathrm{gm}$ wt. Note the breaks in three of the time axes.

action potential. This corresponds approximately to our previous psychophysical measures in humans where the surface was in contact with the skin for about $1 \mathrm{sec}$. The effect of measuring responses over different time intervals is pursued at the end of Results. A few of the fibers had some spontaneous activity; for these it was possible to identify the first stimulusevoked action potential above the spontaneous activity and this was taken as the starting point for analysis.

\section{Responses to stimuli at the receptive field center}

In this sequence the stimuli were applied to the center of the receptive field. The aim was to characterize the responses as a function of the curvature of the stimulus and of the contact force. Fourteen stimuli consisting of the seven curvatures at contact forces of both $10 \mathrm{gm}$ wt and $20 \mathrm{gm} \mathrm{wt}$ were applied in

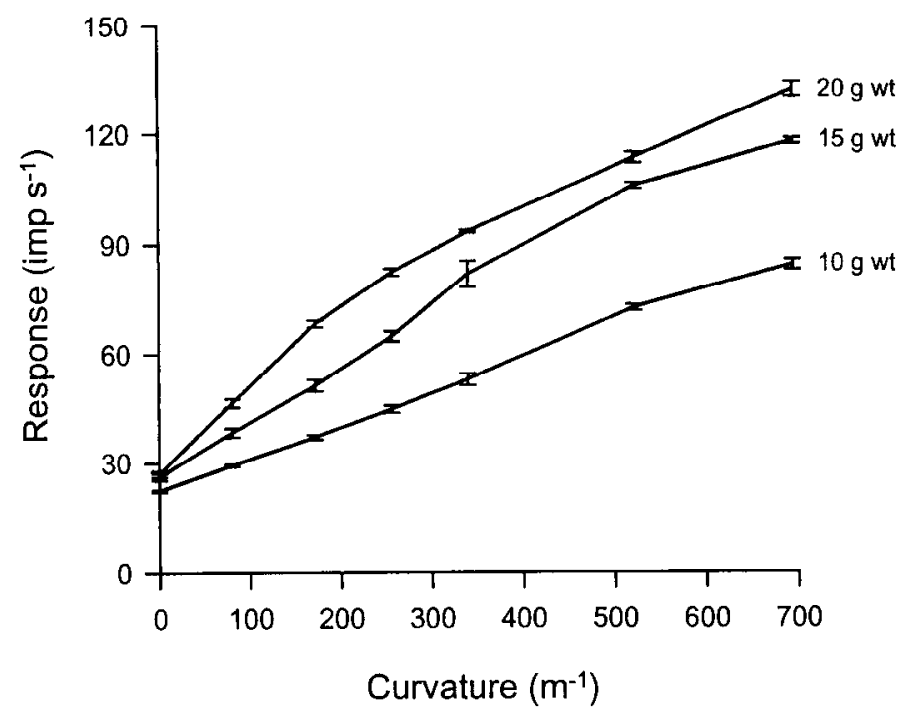

Figure 3. Responses of a single SAI to objects of different curvature applied to the center of the receptive field with different contact forces. The response measure is the total number of impulses occurring during the first second of contact (mean $\pm \mathrm{SEM}, n=8$ ). The eight repetitions for 10 and $20 \mathrm{gm}$ wt occurred at different times but without moving the stimulator; for $15 \mathrm{gm} \mathrm{wt}$ the stimulator was moved to different positions between repetitions. a randomized design. Figure 3 shows the results from a typical SAI. For the 10 and $20 \mathrm{gm}$ wt curves the position of the stimulator was maintained at the center of the receptive field and was not moved. The low standard errors reflect the small variation over time for repeated applications of each combination of curvature and force. Data for the $15 \mathrm{gm}$ wt curve were not part of the random blocks. These were obtained as part of a field study (described below) in which the stimuli were applied at different positions in the receptive field and were located at the center on eight separate occasions. These standard errors are slightly larger than for 10 or $20 \mathrm{gm} \mathrm{wt}$, as they reflect an additional variability caused by repositioning the stimulus. The responses increased with an increase in the curvature of the stimulus and with an increase in contact force. For each curvature-force combination there were eight repetitions at different times, but each repetition had three successive trials. If each trial is treated separately, then $n=24$ and the variability includes that due to adaptation in the relatively short time between the trials (Pubols, 1982). We have averaged the responses for the three trials, eliminating the adaptation effect; thus, $n=8$ and the variability is due to factors other than the intertrial adaptation.

The responses of 16 SAls are shown for a contact force of 10 $\mathrm{gm} w \mathrm{t}$ in Figure 4 (left). All fibers showed an increase in response with an increasc in curvaturc but therc was a considerable spread in the responses of the fibers. At least three factors contributed to the spread. First, it is well known that different SAIs have different sensitivities, giving, in effect, a different scaling factor for each fiber (Sathian et al., 1989). Second, since the pool of fibers was drawn from different fingers and different monkeys, factors like skin and receptor mechanics would have varied. Third, random experimental errors would contribute to the spread. The effect of fiber sensitivity can be removed by normalizing responses. This was done by dividing the responses of each afferent by the average response for that afferent over all seven curvatures. The ratios of the normalizing factors showed that the most sensitive fiber in our sample was 4.3 times as responsive as the least sensitive fiber; the distribution of the normalizing factors is shown in Figure 5. By comparing the responses in Figure 4 (left) with the normalized responses in Figure 4 (right) it can be seen that most of the initial spread was due to differences in fiber sensitivity. The normalized curves 

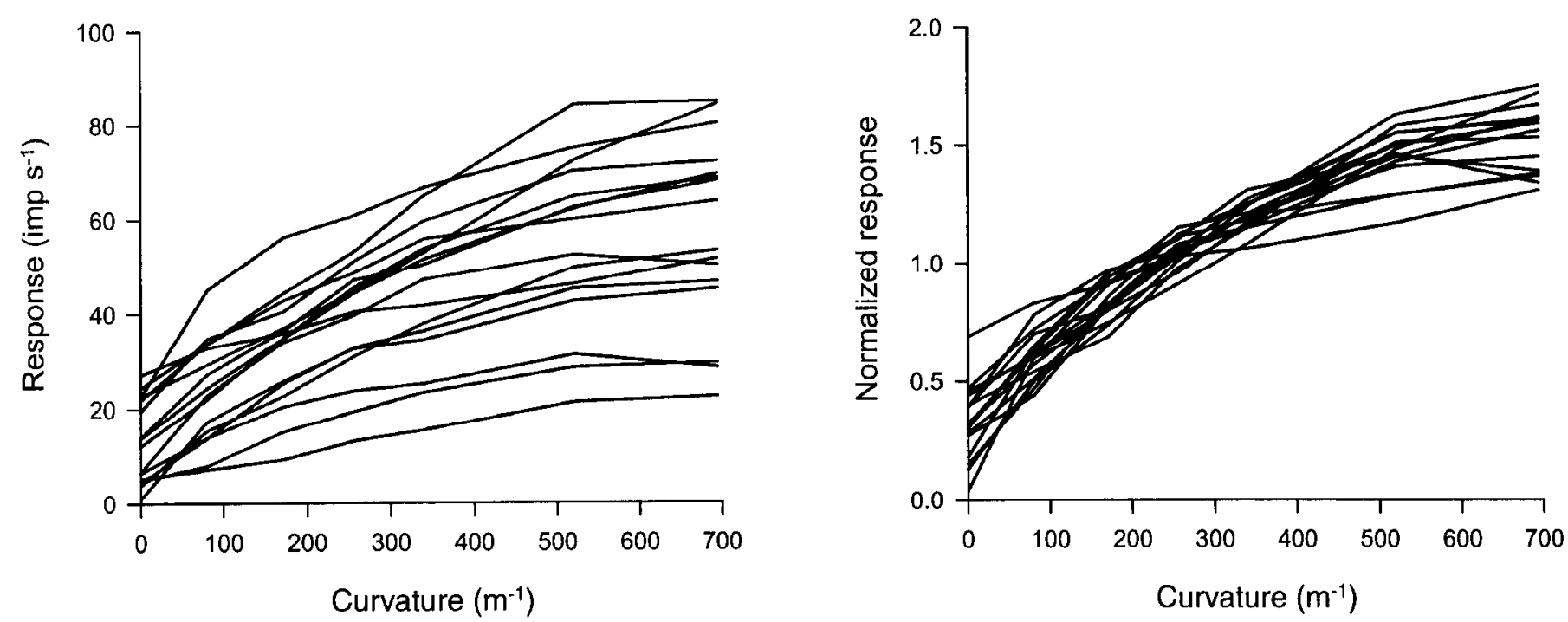

Figure 4. Responses of 16 SAIs to stimuli at the center of the receptive field at a contact force of 10 gm wt. Left, Number of impulses in the first second of contact. Right, Each afferent's responses were normalized by dividing by the average response of that afferent to the seven curvatures.

represent the underlying curvature-response function. Individual fiber responses are defined by this function multiplied by the scaling factor for that fiber.

At contact forces of 15 and $20 \mathrm{gm}$ wt the situation was similar except that the responses were greater at a greater contact force. The curves were normalized as above and Figure 6 (left) shows the means and standard errors for all three contact forces. For 10 and $20 \mathrm{gm}$ wt there are 16 fibers from the randomized study. At $15 \mathrm{gm}$ wt there are 11 fibers used in the field study below, six of which are a subset of the group of 16 fibers. The standard errors reflect variation among fibers excluding differences in sensitivity, namely, variation in skin and receptor mechanics and experimental errors. For each fiber a separate normalizing factor was calculated for each contact force. It is clear from Figure 6 (left) that the underlying curvature-response function is the same at all three contact forces and that a change in contact force merely changes the scaling factor. From the ratios of the normalizing factors, the scaling factors for 10,15 , and $20 \mathrm{gm}$ $w t$ were in the ratio $1: 1.27: 1.52$. An alternative way of displaying the data is to calculate a single normalizing factor for each fiber (the grand mean for that fiber of responses to the 21 forcccurvature combinations). This removes the sensitivity of each fiber but preserves the relative scaling factors for the three forces as seen in Figure 6 (right).

Thus, it is possible to develop a quantitative characterization of the responses of an SAI stimulated at the center of the receptive field. The analysis is done in two stages as follows. The response, $y$, of any SAI is given by the simple expression

$$
y=s k f(x) \text {. }
$$

The constant $s$ is the sensitivity of the fiber and there are three values of the constant $k$, one for each contact force. Inspection of Figure 6 (left) suggests that the curvature-response function $f(x)$ is adequately described by an exponential of the form $a-$ $b \exp (-c x)$. In the first phase, values for $a, b$, and $c$ were obtained by nonlinear regression of the 301 data points in Figure 6 (left). The resulting curve and the data points fitted are shown in Figure 7 (left). The goodness of fit is indicated by $F_{3.297}=1345(\alpha \ll$
0.001). Thus,

$$
y=s k[1.91-1.62 \exp (-0.00243 x)] .
$$

This is used in the second phase to obtain the three values of $k$ by nonlinear rcgression of the data points in Figure 6 (right). The resulting curves and data points fitted are shown in Figure 7 (right). The values of $k$ at 10,15 , and $20 \mathrm{gm}$ wt are 0.792 , 1.01 , and 1.21 , respectively. The goodness of fit for the three curves is given by, for $10 \mathrm{gm} \mathrm{wt}, F_{1,110}=893 ; 15 \mathrm{gm} \mathrm{wt}, F_{1,40}$ $=251$; and $20 \mathrm{gm} \mathrm{wt}, F_{1,110}=930$ (in all cases $\alpha \ll 0.001$ ). The ratios of the values of $k$ for 10,15 , and $20 \mathrm{gm}$ wt are 1:1.28: 1.53 , which agrees well with the ratios calculated above from the normalizing factors. The above relationship between response, curvature, and contact force holds for any SAI.

Nine RAs were studied with the same random sequence of

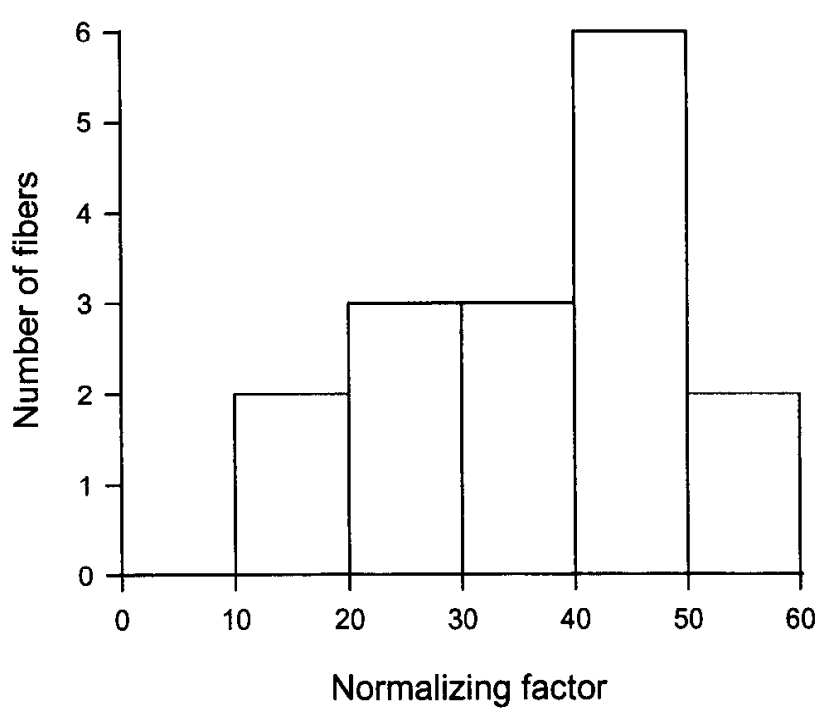

Figure 5. The distribution of sensitivities for the 16 SAIs in our sample is shown by the distribution of the normalizing factors used in Figure 4. 

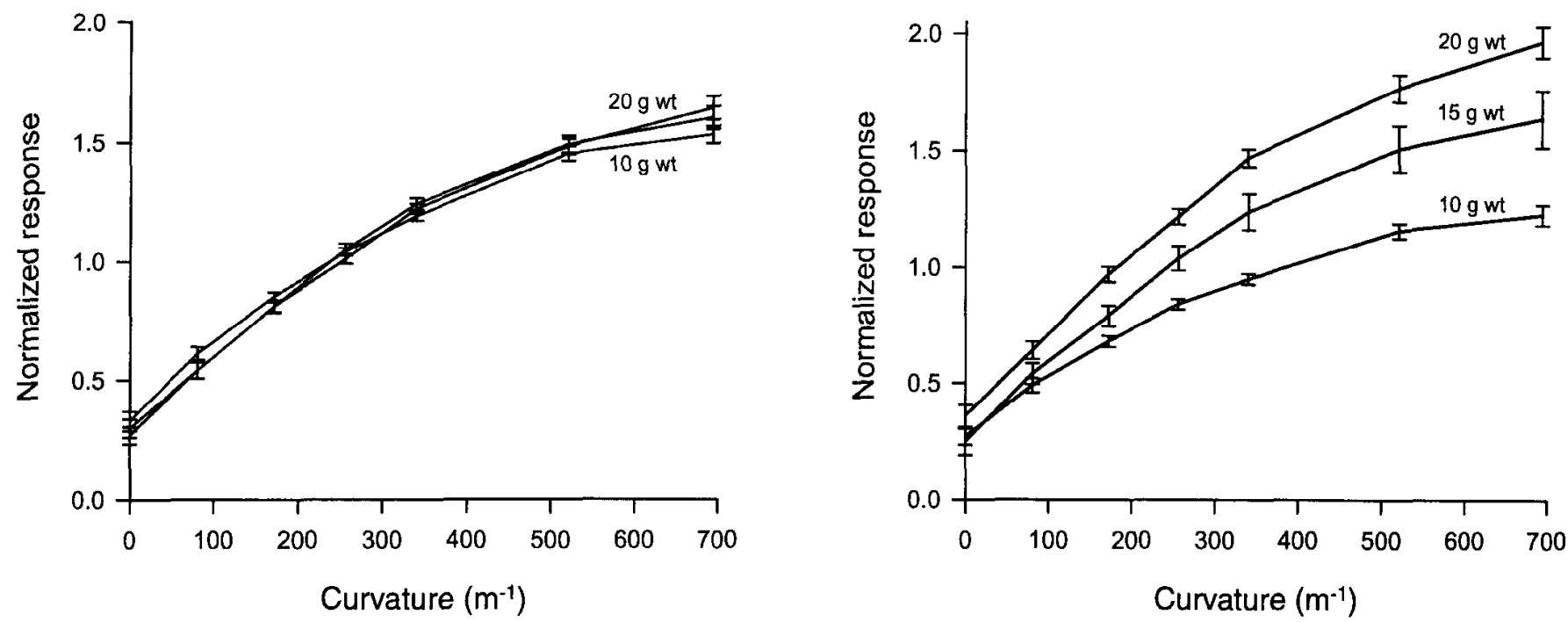

Figure 6. Normalized responses of the SAIs to stimuli at the center of the receptive field (mean \pm SEM). Left, For each fiber a separate normalizing factor is calculated for each contact force. Right, For each fiber a single normalizing factor is calculated for all three contact forces. For 10 and 20 $\mathrm{gm} \mathrm{wt}, n=16$; for $15 \mathrm{gm} \mathrm{wt}, n=11$.

curvatures and forces as used for the SAIs (seven curvatures at 10 and $20 \mathrm{gm} \mathrm{wt}$ ). Two of the RAs did not respond at all to our stimuli. For the remaining seven, the response magnitude was much smaller than for the SAIs. The effect of curvature was small and variable; responses did not consistently increase or decrease with curvature, as shown in Figure 8 . For five of the responding fibers a field study at a contact force of $15 \mathrm{gm} \mathrm{wt}$ (see below) provided data for the curves in Figure 8 (center). In general, there was a slight increase in response with an increase in contact force.

We recorded from 10 PCs. Although the centers of the receptive fields could not be located with certainty, each was clear- ly on a digit and we estimated that the most sensitive point was located on the distal phalanx for four fibers, on the middle phalanx for two fibers, and on the proximal phalanx for four fibers. In each case the stimulus was applied to the center of the fingerpad on the distal phalanx; the rationale for this placement was to see whether any PCs could provide meaningful signals about fingerpad stimulation. Eight of the PCs did not respond at all to our stimuli. The remaining two (one on the distal and one on the proximal phalanx) were spontaneously active. The responses evoked by our stimuli in these two were small and, as revealed by the full random sequence used previously, did not vary consistently with curvature or with contact force.
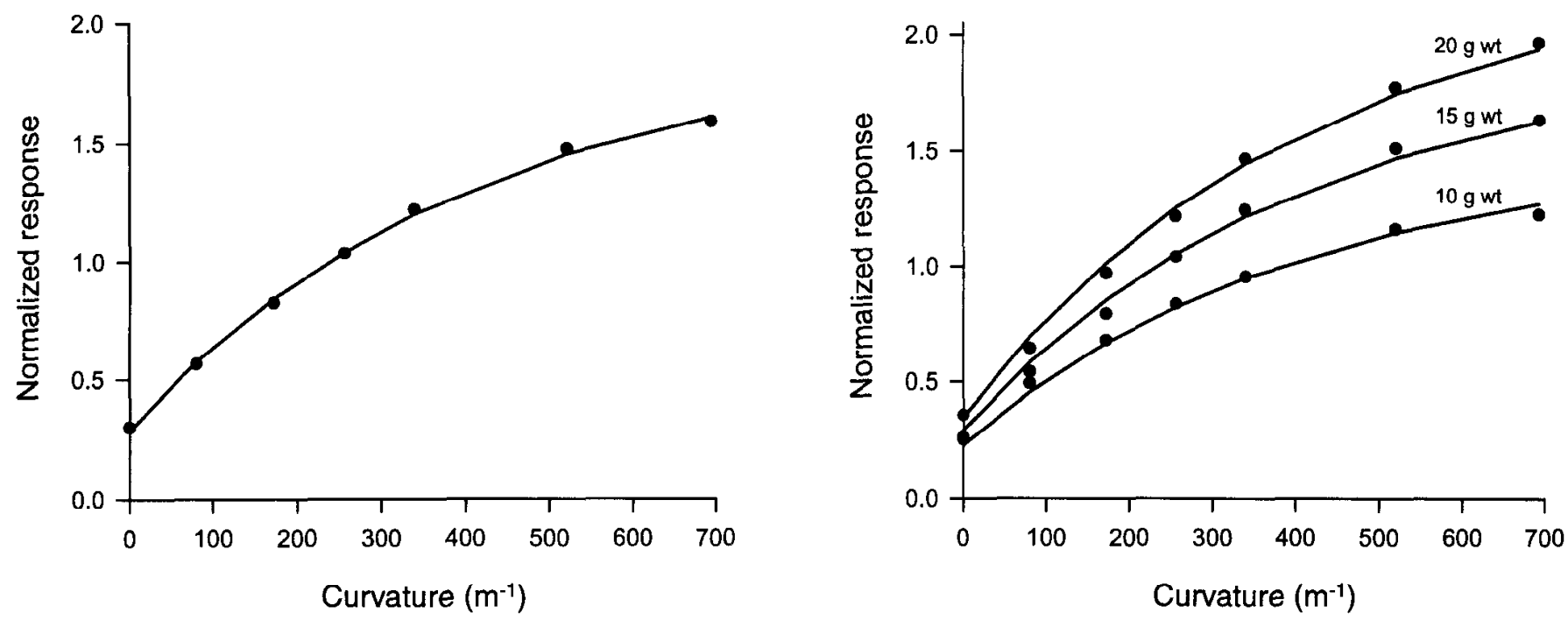

Figure 7. Nonlinear regression of normalized responses to a stimulus at the center of the receptive field. Left, Normalizing factors calculated separately for each contact force. Data points show mean responses at each curvature taken from Figure 6 (left). The curve is the least squares fit of the function $y=a-b \exp (-c x)$ : fitted parameters and coefficients of variation are $a=1.91(2.87 \%), b=1.62(3.09 \%), c=2.43 \times 10^{-3}(6.84 \%)$, $n=301$. Right, The same normalizing factor for all contact forces. Data points show mean responses at each curvature and each contact force taken from Figure 6 (right). Curves are least squares fits of the function $y=k[1.91-1.62 \exp (-0.00243 x)]$ : fitted parameters and coefficients of variation are, for $10 \mathrm{gm} \mathrm{wt}, k=0.792(1.33 \%), n=112 ; 15 \mathrm{gm} \mathrm{wt}, k=1.01(2.49 \%), n=42 ; 20 \mathrm{gm} \mathrm{wt}, k=1.21(1.30 \%), n=112$. 


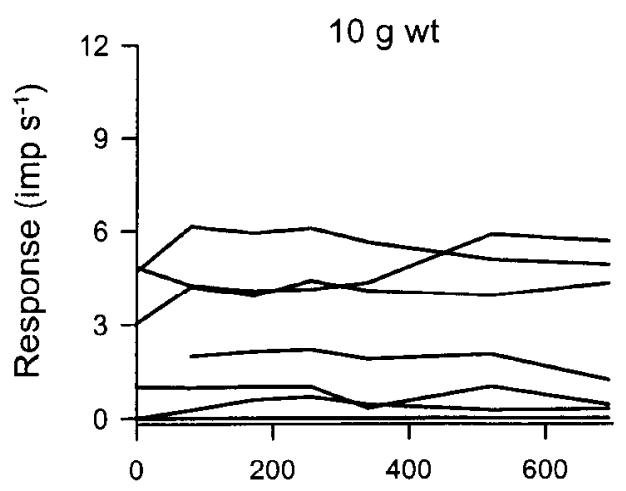

Curvature $\left(m^{-1}\right)$

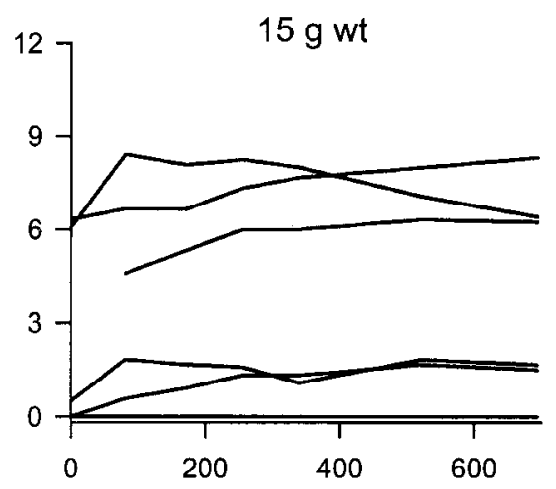

Curvature $\left(\mathrm{m}^{-1}\right)$

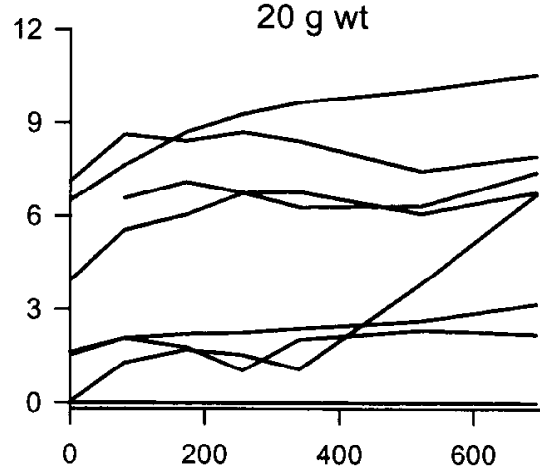

Curvature $\left(\mathrm{m}^{-1}\right)$

Figure 8. Responses (number of impulses in $1 \mathrm{sec}$ of contact) of RAs stimulated at the center of the receptive field. Two of the afferents did not respond at all. Data at 10 and $20 \mathrm{gm} w \mathrm{wt}$ are from a complete random sequence, $n=9$. For five of the seven responding afferents, data gathered during a field study at $15 \mathrm{gm}$ wt are shown, $n=7$ (five responding and two nonresponding afferents).

\section{Responses to stimuli at different points in the receptive field}

In this series the stimulator was placed at various points in the receptive field and the seven curved surfaces were applied with a contact force of $15 \mathrm{gm}$ wt. At each point the order of presentation of the surfaces was random and each surface was applied in a single sequence of three trials; each trial consisted of $4 \mathrm{sec}$ of contact followed by $4 \mathrm{sec}$ of no contact. The responses of a typical SAI are illustrated in Figure 9 at points, separated by $0.5 \mathrm{~mm}$, along two lines through the center of the receptive field, one parallel to the long axis of the finger and the other at right angles to it. For the most curved surfacc $\left(694 \mathrm{~m}^{-1}\right)$, responses decreased as the surface moved away from the receptive field center either in the $y$-direction or in the $\mathrm{x}$-direction. For the less curved surfaces the same was true but the profiles differed in two respects. First, for smaller curvatures the response at the center was smaller. Second, for smaller curvatures the decrease in response with increasing distance from the center of the receptive field was less rapid. These trends continued with de- creasing curvature. As expected, for a flat surface there was no significant effect of distance.

The responses of 13 SAIs to the surface with a curvature of $340 \mathrm{~m}^{-1}$ are shown in Figure 10 (left). As expected, the responses are greater for some afferents than for others. When the responses are normalized to remove the differences due to variation in sensitivity of the afferents, the curves (Fig. 10, right) are similar for all fibers. Thus, the underlying variation of response with position is the same for all SAIs, and their different sensitivities simply result in a different scaling factor. Figure 10 illustrates this for the curvature $340 \mathrm{~m}^{-1}$ applied at points on the line $x-0$, but it was true for all the positions in the receptive field and all the curvatures examined. Hence, the effect of the position of the stimulus in the receptive field and the effect of the curvature of the surface can be described by profiles consisting of the averaged normalized responses across our sample of SAIs. For example, the average of the curves in Figure 10 (right) would show the profile for a curvature of $340 \mathrm{~m}^{-1}$ along the $y$-axis $(x-0)$. These profiles are three-dimensional with the
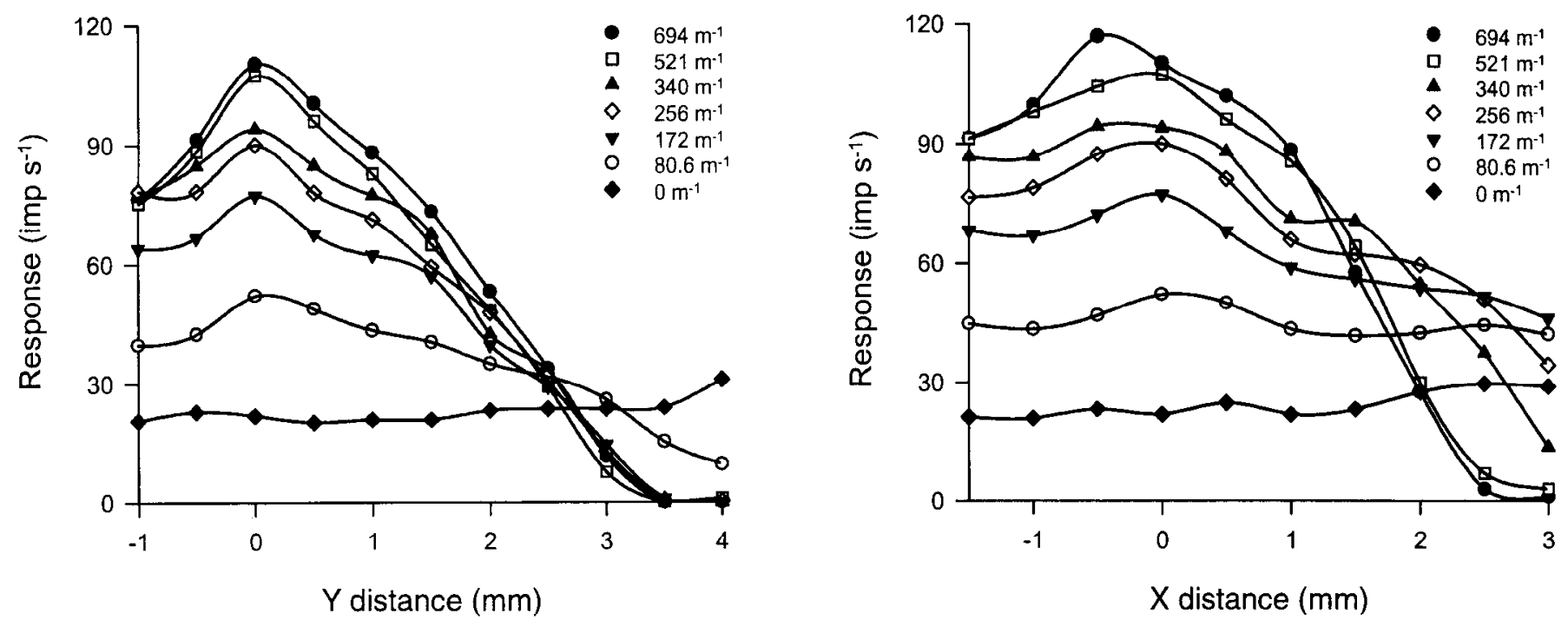

Figure 9. Responses (number of impulses in $1 \mathrm{sec}$ ) of an SAI to the seven curvatures as a function of distance from the receptive field center. The points stimulated were separated by $0.5 \mathrm{~mm}$ and lay on a line through the center of the receptive field parallel to (left) or at right angles to (right) the long axis of the finger. These lines are defined as the $y$-axis $(x=0)$ and $x$-axis $(y=0)$, respectively; the center of the receptive field is the origin $x=0, y=0$; see Figure 1 . 

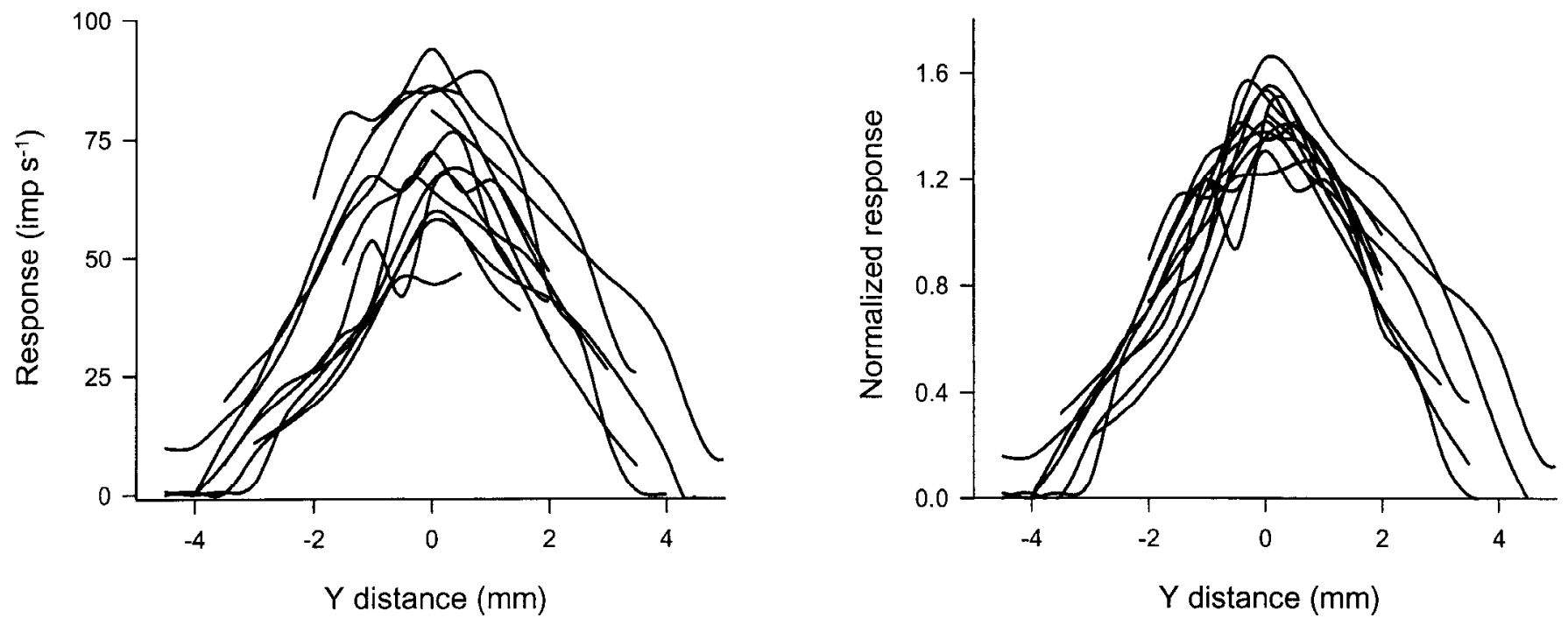

Figure 10. Responses of 13 SAIs to a surface of curvature $340 \mathrm{~m}^{-1}$ as a function of distance from the receptive field center. Points, separated by $0.5 \mathrm{~mm}$, lay along the line $x=0$ (parallel to the long axis of the finger). Left, Responses are the number of action potentials elicited during the first second. Right, To remove variation due to different fiber sensitivities, responses have been normalized by dividing each fiber's responses by the mean response for that fiber.

response being a function of both the $x$ - and the $y$-position in the receptive field, and the profile is different for each curvature. This is difficult to illustrate completely but Figure 11 shows the main features of the profiles. Figure $11 A$ shows the profiles along the $y$-axis $(x=0)$ for the seven curvatures. As the curvature of the surface decreased, the height of the profile decreased and it became broader or less peaked. This effect was demonstrated for single fiber responses in Figure 9. In Figure $11 B$ the profile is illustrated for the curvature $694 \mathrm{~m}^{-1}$ for lines parallel to the long axis of the finger at successively increasing distances from the center of the receptive field. The line $x=0$ is through the center; the line $x=0.5$ is the average of points on the two lines $0.5 \mathrm{~mm}$ from the center $(x=+0.5 \mathrm{~mm}$ and $x=-0.5 \mathrm{~mm})$, and so on. Figure $11 B$ depicts the rapid falloff of the profile in two directions (i.e., the $\mathrm{x}$ - and $\mathrm{y}$-directions on the finger) for a curvature of $694 \mathrm{~m}^{-1}$. At any value of $x$, response declines rapidly as the surface moves away from the center of the receptive field in the y-direction and, conversely, at any value of $y$, response declines rapidly as the surface moves away from the center of the receptive field in the $\mathrm{x}$-direction. Figure $11 \mathrm{C}$ shows a similar display of the profile for the curvature of $340 \mathrm{~m}^{-1}$. It

Table 1. Regression of $z=a \exp \left(-b x^{2}-c y^{2}\right)$

\begin{tabular}{cllll}
$\begin{array}{c}\text { Curva- } \\
\text { ture }\end{array}$ & $a$ & $b$ & $c$ & $F$ \\
\hline 694 & 1.79 & 0.279 & 0.191 & 814 \\
521 & 1.67 & 0.229 & 0.178 & 924 \\
340 & 1.35 & 0.121 & 0.142 & 775 \\
256 & 1.12 & 0.0670 & 0.112 & 572 \\
172 & 0.860 & 0.0199 & 0.0695 & 279 \\
80.6 & 0.549 & $2.3 \times 10^{-10}$ & 0.0151 & 14.4 \\
0 & 0.317 & $4.3 \times 10^{-10}$ & $2.0 \times 10^{-10}$ & $3.61 \times 10^{-4}$
\end{tabular}

Data represent normalized response $z$ as a function of position $(x, y)$ of the stimulus on the fingerpad. For each curvature, $n=532$. For the surfaces with curvatures from 694 to $80.6 \mathrm{~m}^{-1}$ the significance levels of the $F_{3.528}$ values are $\alpha \ll 0.001$; for $0 \mathrm{~m}^{-1}$ the regression is not significant. is clear from Figure $11 A$ that in the plane $x=0$ the profile for $340 \mathrm{~m}^{-1}$ is broader than that for $694 \mathrm{~m}^{-1}$ and this is reflected in Figure $11 C$, where the profile at $x=0$ has a smaller height than in Figure $11 B$ and where the reduction with increasing $y$-distance from the center of the receptive field is less rapid. In addition, by comparing Figure $11, B$ and $C$, it is seen that the three-dimensional profile for a curvature of $340 \mathrm{~m}^{-1}$ falls off less rapidly both in the $\mathrm{x}$-direction and in the $\mathrm{y}$-direction than does the profile for a curvature of $694 \mathrm{~m}^{-1}$. This trend continues and becomes more obvious with decreasing curvature, as seen in Figure $11 \mathrm{D}$. For clarity, standard errors are not shown in Figure 11, but the fiber-to-fiber variability is clearly shown in Figure 10 (right), which is typical of all curvatures and all positions.

A quantitative comparison of the three-dimensional profiles is facilitated by fitting a function to the data points. The curves illustrated above suggest a function of the form

$$
z=a \exp \left(-b x^{2}-c y^{2}\right)
$$

where $z$ is the normalized response and $x$ and $y$ are the positions on the fingerpad as defined in Figure 1. This assumes symmetry about the $x$ - and $y$-axes but allows different rates of decay in the $x$ - and $y$-directions. For cach of the seven curvatures the above function was fitted, by nonlinear regression, to the data points at all positions in the receptive field; $n=532$ for each profile. Table 1 shows the values of the constants $a, b$, and $c$ as well as measures of the goodness of fit. For all curvatures except $0 \mathrm{~m}^{-1}$, the high $F$ values attest to the suitability of the function and the close fit to the data. For the flat surface the low $F$ value indicates that the data do not differ significantly from the mean value, as should be the case. Figure 12 compares the data (average of the normalized responses of the SAIs) with the fitted functions; Figure 12 (left) compares all seven curvatures for the line $x=0$ and Figure 12 (right) compares all values of $x$ and $y$ for a curvature of $694 \mathrm{~m}^{-1}$. Figure 13 shows the three-dimensional profile for three different curvatures illustrating clearly the decrease in profile height and the increase in profile width with a decrease in the curvature of the stimulus. The values of 
A. $\quad \mathrm{X}=0$

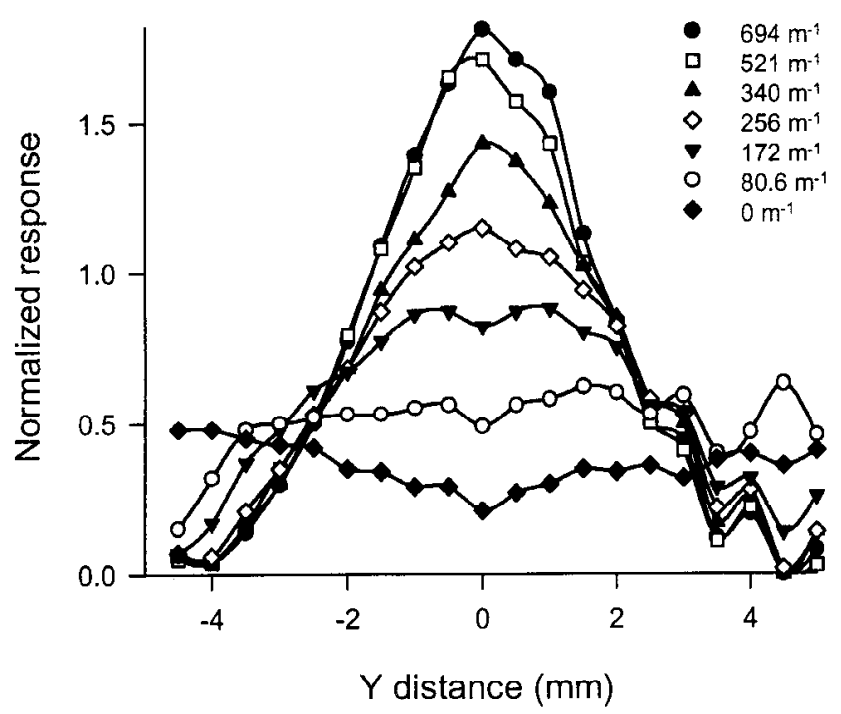

C. $\quad 340 \mathrm{~m}^{-1}$

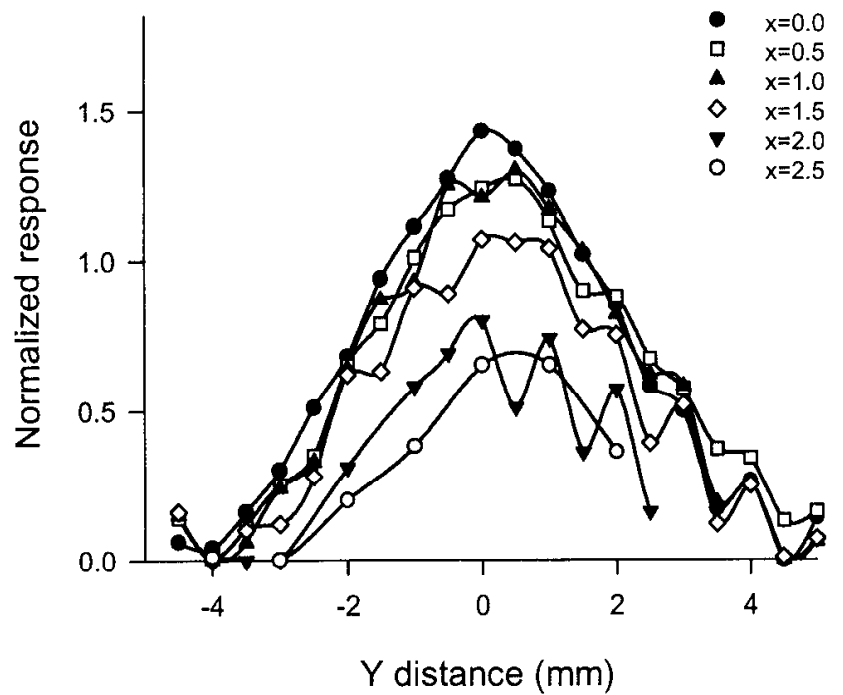

B. $694 \mathrm{~m}^{-1}$

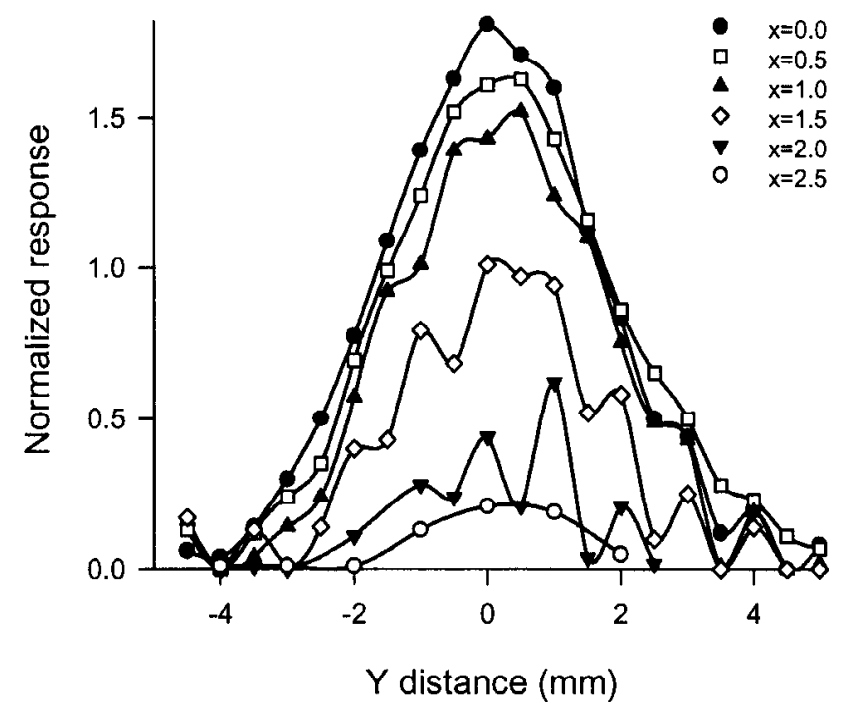

D. $256 \mathrm{~m}^{-1}$

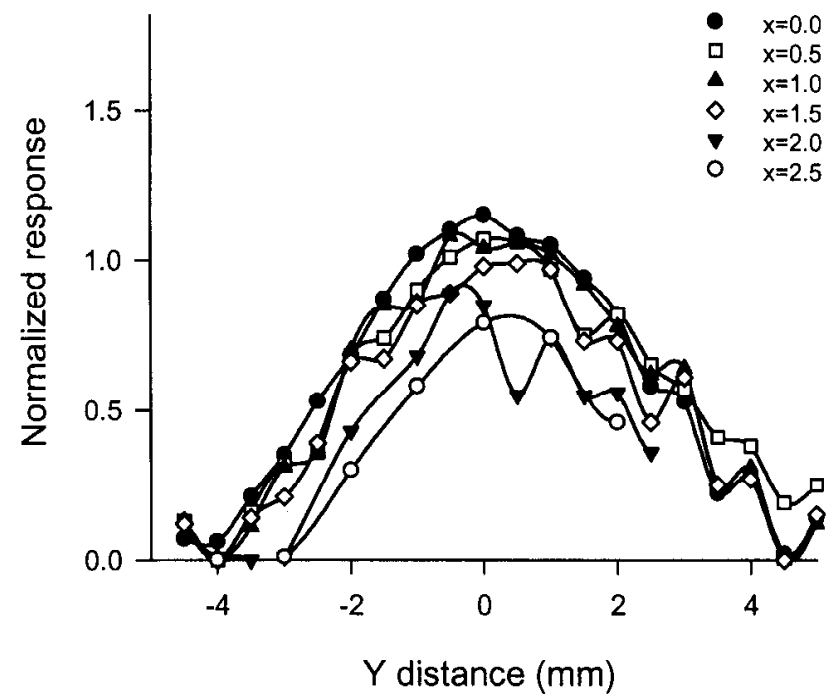

Figure 11. Profiles formed by averaging normalized responses across our sample of SAIs. $A$, Profiles along the line through the center of the receptive field (y-axis) for the seven curvatures. $B-D$, Profiles for curvatures 694,340 , and $256 \mathrm{~m}^{-1}$, respectively. Curves show profiles along lines parallel to the long axis of the finger at distances of $0,0.5,1,1.5,2$, and $2.5 \mathrm{~mm}$ from the center of the receptive field. The profile at $x=1 \mathrm{~mm}$ is the average of the normalized responses of the SAIs at $x=+1 \mathrm{~mm}$ and $x=-1 \mathrm{~mm}$ (i.e., $1 \mathrm{~mm}$ from the center of the receptive field on either side of the $y$-axis) and similarly for the other lines.

the constant $a$ in Table 1 quantify this decrease in profile height, and the values of the constants $b$ and $c$ quantify the increase in profile width. Constants $b$ and $c$ are usually not equal, indicating a difference in the two orthogonal directions on the finger (see Discussion).

As explained in Materials and Methods, for each fiber there were different practical limitations on the spatial extent over which the field profiles could be measured. The distal phalanx of the monkey is smaller than that of the human and approaches the radius of the largest sphere $(12.4 \mathrm{~mm})$. Thus, we were particularly careful to ensure that all positions studied were on a sufficiently flat part of the fingerpad; the contact area for all spheres was on this flat region and the direction of force was approximately orthogonal to the skin. The resulting limits on the maximum excursions in the $\mathrm{x}$ - and $\mathrm{y}$-directions depended on the location of the center of the receptive field. In Figures 10-13, data are averaged for 13 SAIs but the number of afferents contributing to each point are different, with fewer fibers contributing to the more distant points. For each curvature the complete profile contains 532 points from the 13 fibers. To assess long-term stability and consistency of responses, we repeated measurements at the center of the receptive field $(x=0$, $y=0$ ) several times as part of the field study. These responses were always highly consistent. This is illustrated by the $15 \mathrm{gm}$ wt line in Figure 3, where the eight repetitions during the field study occurred over $8.5 \mathrm{hr}$ and show low standard errors.

\section{Effect of contact force on response profiles}

In a previous section, the responses to stimuli at the center of the receptive field were compared at contact forces of 10,15 , 

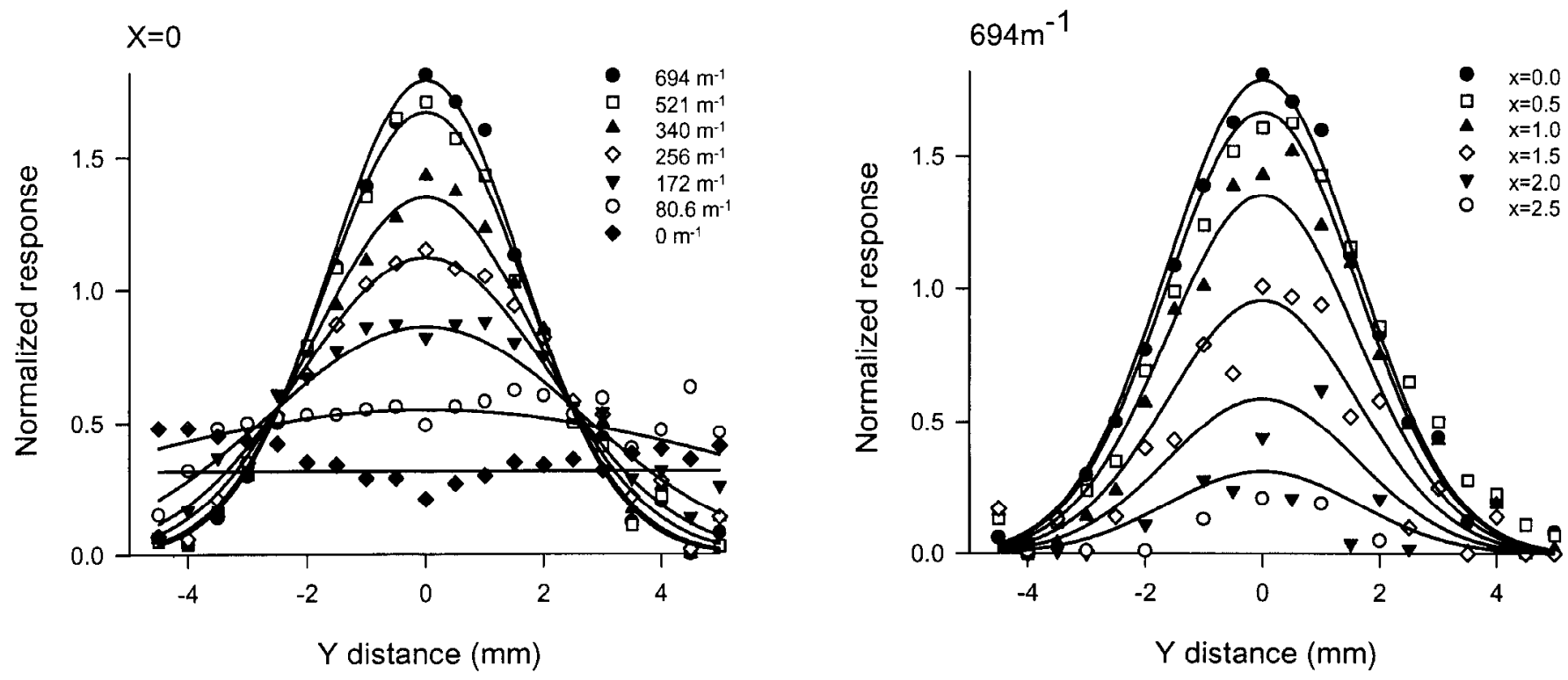

Figure 12. Comparison of experimental data and the fitted functions $z=a \exp \left(-b x^{2}-c y^{2}\right)$. Data points show the average normalized responses of the SAIs and lines show the fitted functions. Left, Responses for all seven curvatures along the line through the center of the receptive field $(x$ $=0)$. Right, Responses for a curvature of $694 \mathrm{~m}^{-1}$ for lines at successive $0.5 \mathrm{~mm}$ intervals from the center of the receptive field.

and $20 \mathrm{gm}$ wt. In this section, the effect of contact force on response profiles was studied by recording responses at points, separated by $1.0 \mathrm{~mm}$, along the line through the center of the receptive field parallel to the long axis of the finger $(x=0)$. At each point four contact forces $(10,15,20$, and $25 \mathrm{gm} \mathrm{wt}$ ) were used. Two surfaces were tested, one of the more curved (521 $\left.\mathrm{m}^{-1}\right)$ and one the least curved $\left(172 \mathrm{~m}^{-1}\right)$. The stimulator was positioned at successive points along the line; at each point the $172 \mathrm{~m}^{-1}$ surface was applied at contact forces of $10,15,20$, and $25 \mathrm{gm}$ wt in order followed by the $521 \mathrm{~m}^{-1}$ surface with the same order of forces. The whole procedure was then repeated usually seven times. Figure $14, A$ and $B$, shows the responses of a typical SAI. All seven SAIs examined behaved similarly and in Figure 14, $C$ and $D$, the responses of the seven fibers have been combined by first normalizing them to remove the effect of the sensitivities of the fibers and then averaging. In general, for both surfaces an increase in contact force increased the response. However, it is clear from Figure 14 that, with increasing force, the profiles are not simply scaled upward. There is certainly an upward scaling but, as the contact force increases from $10 \mathrm{gm}$ wt to $25 \mathrm{gm}$ wt, there is an additional progressive widening of the profiles. This is very obvious if the profiles are normalized and overlaid to check for scaling (not illustrated here). As expected, the profiles coalesce toward the two ends. The curves in Figure 15, replotted from the data in Figure $14 C$, show the above effects with a different emphasis. At the receptive field center $(y=0)$ the force-response curve is concave down, but as the stimulus moves progressively away from the center, the curves flatten. We do not have sufficient data for a comprehensive analysis of this aspect of force effects.

$694 \mathrm{~m}^{-1} \quad 340 \mathrm{~m}^{-1}$
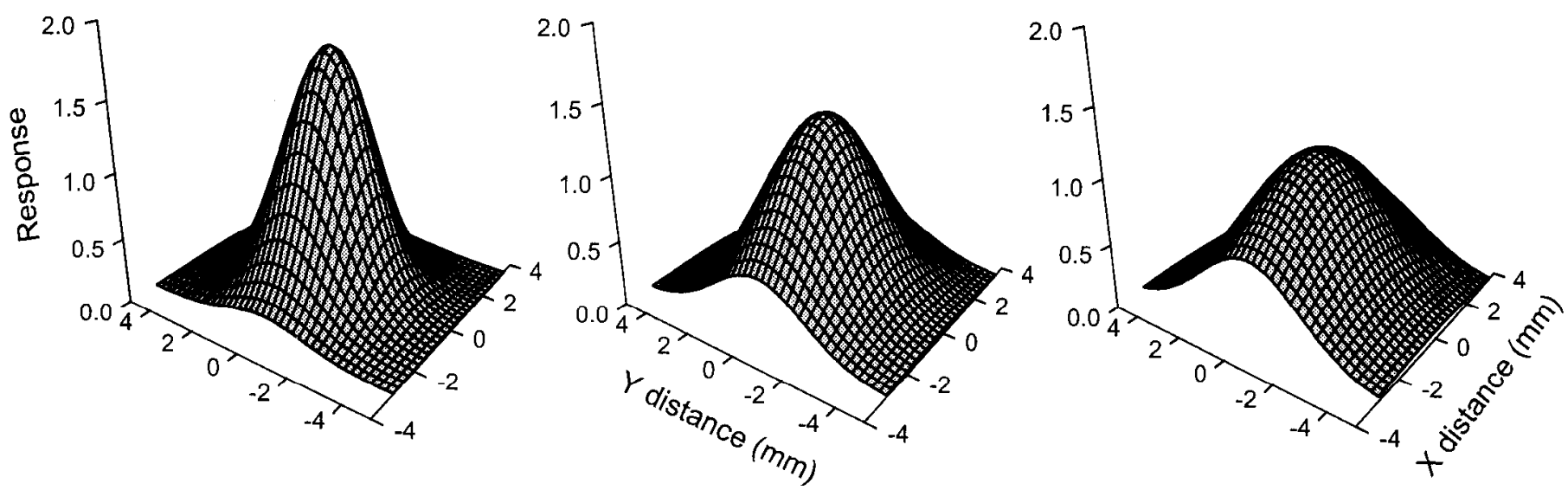

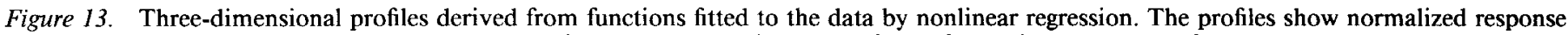

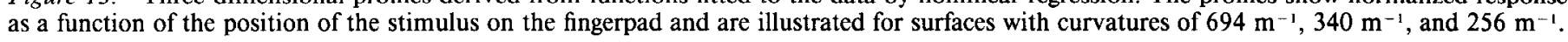




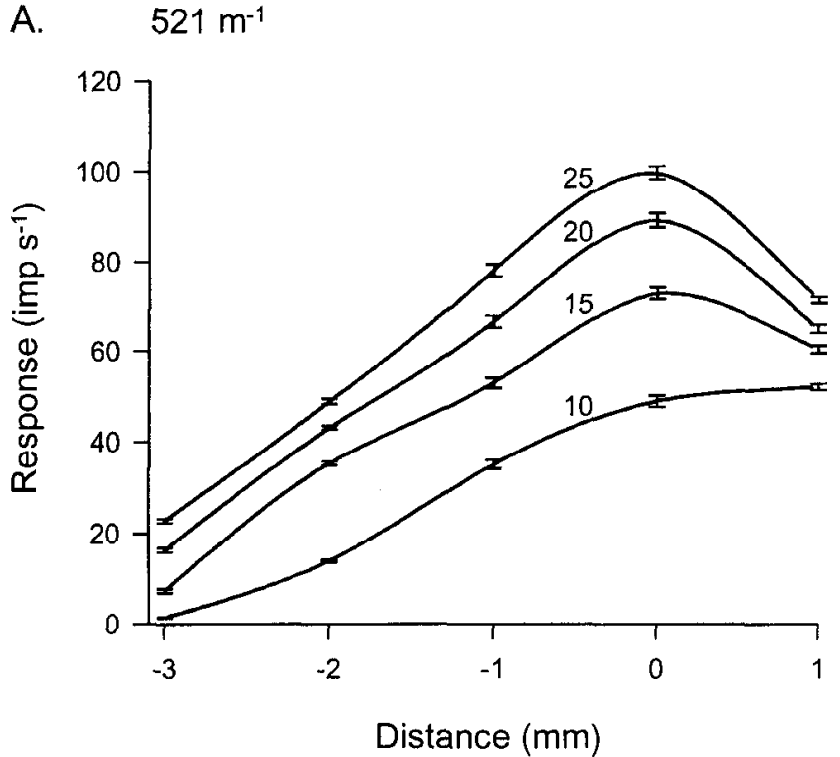

C. $\quad 521 \mathrm{~m}^{-1}$

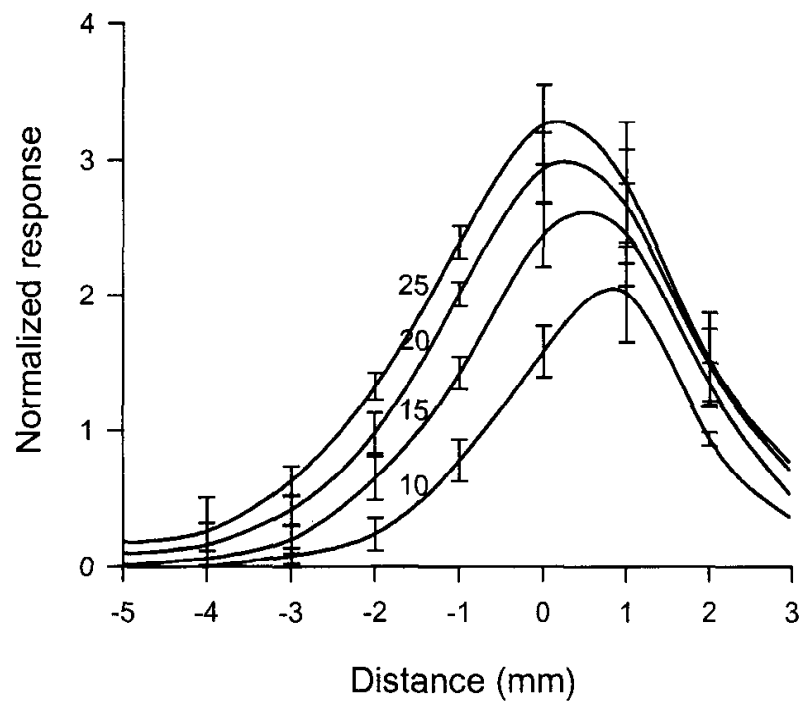

B. $\quad 172 \mathrm{~m}^{-1}$

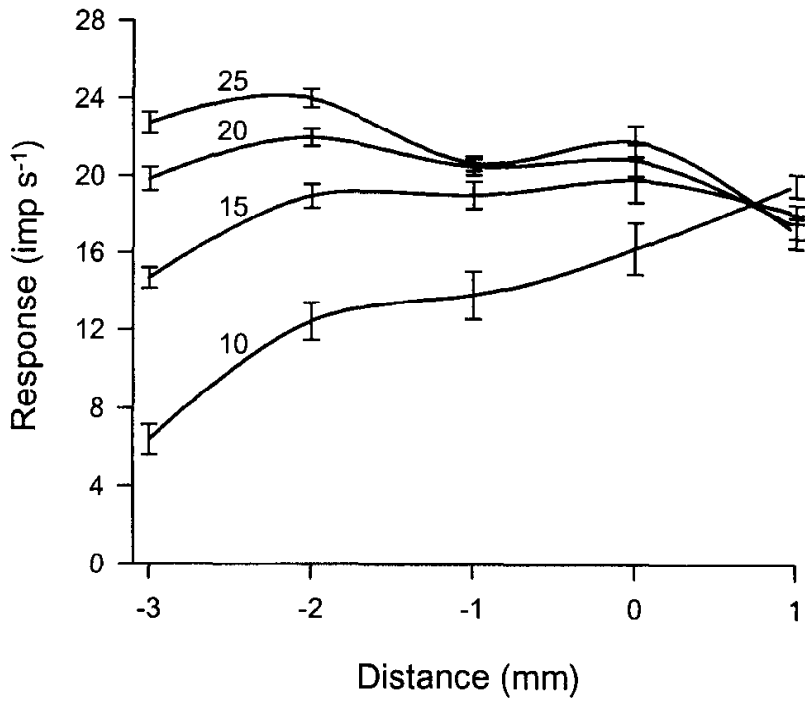

D. $\quad 172 \mathrm{~m}^{-1}$

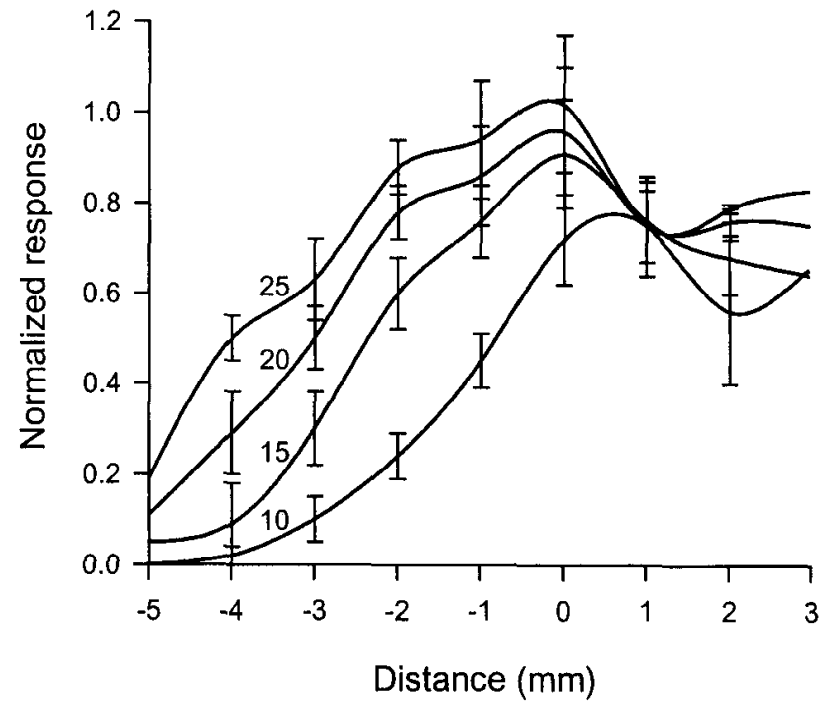

Figure 14. Response profiles at four contact forces for points along a line through the center of the receptive field and parallel to the long axis of the finger (y-axis). Surface curvature was $521 \mathrm{~m}^{-1}(A$ and $C)$ or $172 \mathrm{~m}^{-1}(B$ and $D)$. $A$ and $B$, Responses (mean \pm SEM) for a single SAI, $n=8$. $C$ and $D$, Responses of seven fibers have been combined by first normalizing each fiber's responses (mean $\pm \operatorname{SEM}, n=7$ ).

\section{Different response measures}

For all the data shown so far the response measure has been the number of action potentials evoked during the first second. We have also analyzed some of the data using a variety of integration times for the response measure. In all cases, the time over which impulses were summed had little effect on the results. For example, for stimuli at the center of the receptive field, the shape of the curvature-response function was unchanged and, for stimulation at different points in the receptive field, the shape of the response profile remained constant. This is illustrated in Figure 16 (left), which shows the responses of an SAI as a function of the curvature of a stimulus at the receptive field center; the time over which action potentials were summed to calculate the response was varied from 0.5 to $3.5 \mathrm{sec}$. As before, contact was signaled by the commencement of evoked action potentials. An increase in integration time resulted in an increase in response.
When the curves at each integration time were normalized by dividing the responses by the mean response at that integration time, the resulting curves overlaid one another, as seen in Figure 16 (right). Thus, changing the integration time did not affect the form of the function (in this case the shape of the curvatureresponse function) and simply introduced a scaling constant. For their stimuli, LaMotte and Srinivasan (1993) found an increase in the slope of the curvature-response function for longer integration times. An exhaustive analysis on this point has not been done and it is likely that some SAIs will show more change than others.

\section{Discussion}

\section{Responses of individual afferents}

The first and most direct way of interpreting our data is in terms of the response properties of individual peripheral nerve fibers. We have quantified the relationship between the responses of 


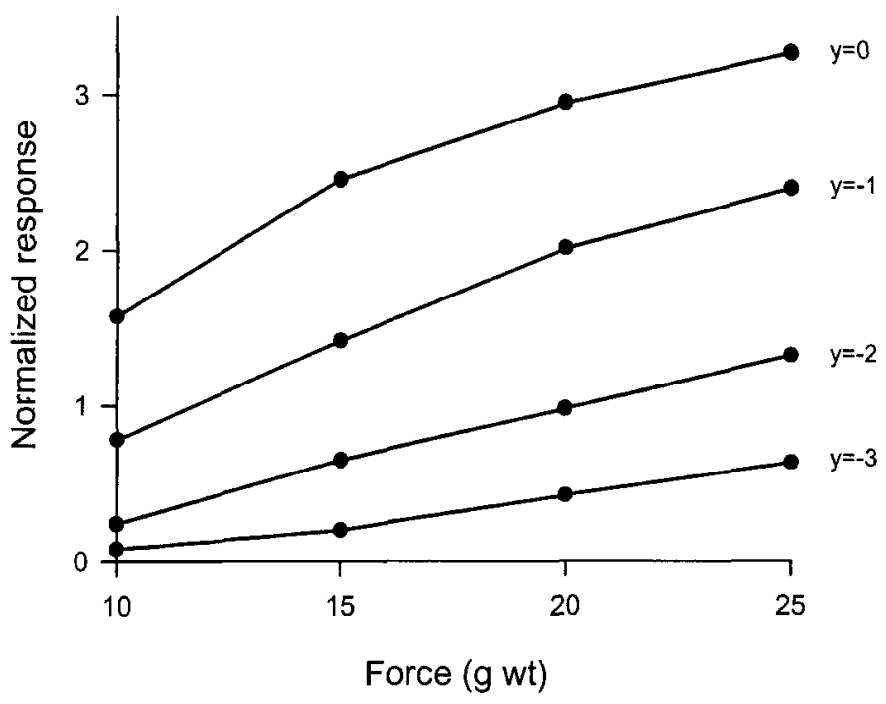

Figure 15. Effect of distance from the center of the receptive field on force-response functions. Data have been replotted from Figure $14 C$. The stimulus, curvature $521 \mathrm{~m}^{-1}$, was placed at distances of $0,1,2$, or $3 \mathrm{~mm}$ from the receptive field center along the $y$-axis.

an SAI and the curvature of a stimulus centered in the receptive field. Starting from a flat surface, responses increased rapidly as curvature increased, and further increases in curvature resulted in progressively diminishing increases in response. The response also increased as the contact force between the stimulus and the fingerpad increased. All SAIs behaved in the same way but had different sensitivities. The response of any SAI is well described by the function

$$
s k[1.91-1.62 \exp (-0.00243 x)],
$$

where $x$ is the curvature of the stimulus, $s$ is the sensitivity of the afferent, and the constant $k$ reflects the effect of contact force and is $0.792,1.01$, and 1.21 for contact forces of 10,15 , and $20 \mathrm{gm} w \mathrm{w}$, respectively. The factors that determine these char- acteristics are the nature of the skin mechanics and of the receptor transduction mechanisms. Unfortunately, there have been few quantitative studies of these mechanical factors. In broad terms, our data are consistent with the continuum mechanics model of Phillips and Johnson (1981b) and the waterbed model of Srinivasan (1989). Our data provide further direct measurements for refining these or alternative models. In terms of previous neural recordings from peripheral nerves, comparison of responses at the two extremes of the above curvature-response function (flat and highly curved) corresponds to the reported edge enhancement effect (Vierck, 1979; Phillips and Johnson, 1981a; Johansson et al., 1982). Our data are also consistent with Srinivasan and LaMotte's (1987) results on indented sinusoidally shaped steps where responses increased with an overall increase in curvature, and with LaMotte and Srinivasan's (1993) experiments with indented cylinders where responses increased when curvature increased in one direction.

By systematically varying the position of the curved surfaces within the receptive field, we have characterized the spatial response profiles of the SAIs. For any particular curvature, all SAIs had similar profiles that differed only by a scaling factor equivalent to the sensitivity of the afferent. The shape of the profile reflected the shape of the stimulus. As the curvature of the stimulus decreased, the profile decreased in height and became broader. Responses were well described by the function

$$
a \exp \left(-b x^{2}-c y^{2}\right) \text {, }
$$

where $x$ and $y$ are the coordinates of the position of the stimulus in the receptive field and the constants $a, b$, and $c$ change as the curvature of the stimulus changes. As curvature increases, $a$ incrcases (this is the curvaturc-rcsponse function at the center of the receptive field discussed above) and $b$ and $c$ increase. These characteristic profiles are also determined by the mechanics of the skin and receptors. We know of no model that has been tested on data resembling ours. Our results provide specific measures of the spatial characteristics of SAI receptive fields that must be explained by models developed in the future. Since RA responses to our stimuli were small and did not change
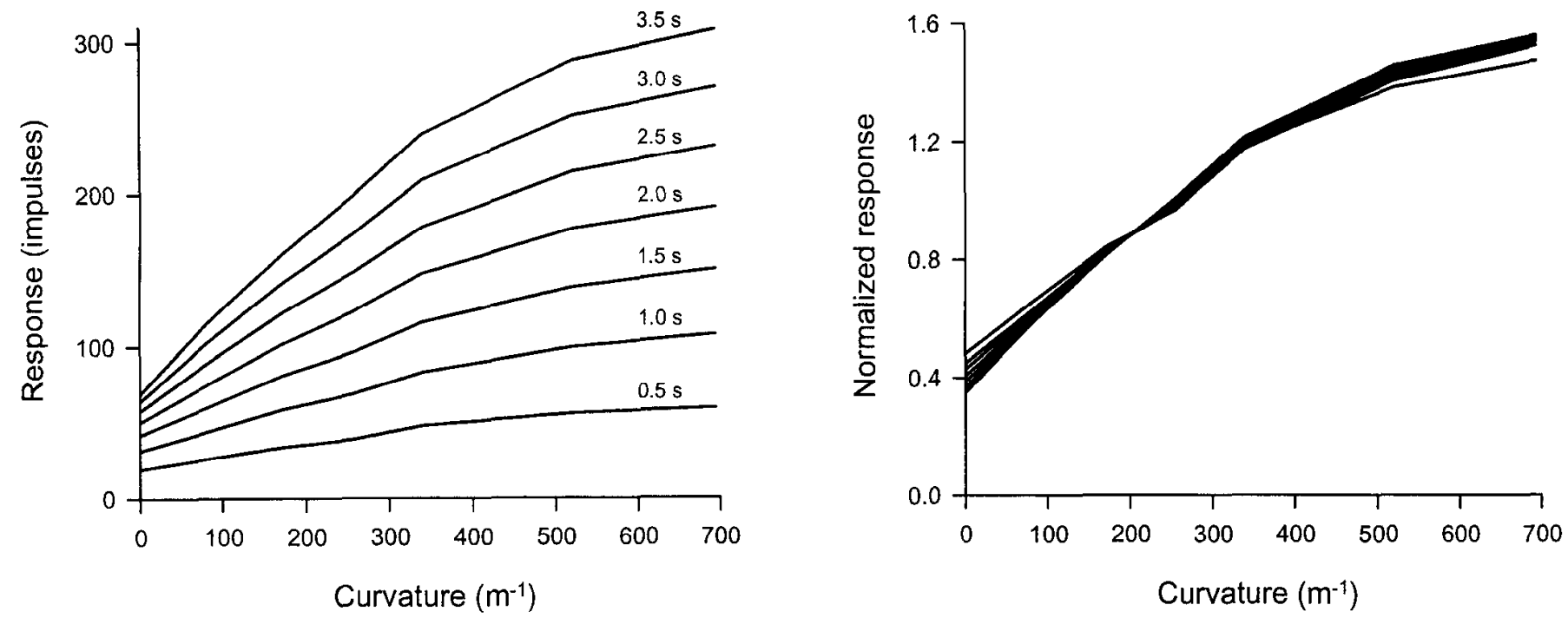

Figure 16. Responses as a function of the curvature of surfaces applied to the center of the receptive field of an SAI. Contact force was 20 gm wt. The response measure is the total number of action potentials during a window commencing at the first stimulus-evoked action potential. Window widths range from 0.5 to $3.5 \mathrm{sec}$ in $0.5 \mathrm{sec}$ increments. Left, Actual responses. Right, Normalized curves; each curve in the left panel has been normalized by dividing each response by the average response for that curve. 
consistently with changes in the curvature of the stimulus, we did not measure spatial response profiles for RAs.

\section{Population responses}

The second way in which our data can be interpreted is by extension to population responses (Johnson, 1974; Goodwin and Pierce, 1981; Cohen and Vierck, 1993b). This step is essential in using our results to explain human perception. When a curved surface contacts the skin, a population of afferents innervating the skin in the region of the surface will be activated. The surface will be located at different distances from the receptive field centers of different afferents. Thus, the profile of responses across the SAI population will resemble the profile described above for single afferents. That is, for highly curved objects (small radii) the population profiles will be high and narrow. As the curvature decreases (radius increases), the profile becomes lower and broader so that the shape of the profile reflects the shape of the stimulus. If all the SAIs had identical properties, then the population profile would be identical to the single afferent profiles. Our data show that as the curvature of the stimulus changes, the shape of the profile changes correspondingly. This explains how humans are able to scale and discriminate the curvature of objects in contact with the fingerpad using only cutaneous receptors (LaMotte and Srinivasan, 1987a; Srinivasan and LaMotte, 1987; Goodwin et al., 1991; Goodwin and Wheat, 1992a). An increase in contact force results in an upward scaling of the response profiles, explaining how humans are able to scale the contact force using cutaneous afferents (Goodwin and Wheat, 1992b). For a single afferent, responses increase with either an increase in curvature or an increase in contact force so that the information provided by that afferent alone is ambiguous. However, when the population of afferents is viewed, a change in the shape of the stimulus changes the shape of the profile whereas a change in the contact force scales the profile. Thus, it is a simple matter to extract both the shape of the stimulus and the contact force from the SAI population. This explains how, with passive touch, humans can scale for both shape and contact force when both arc changing randomly (Goodwin and Wheat, 1992b). LaMotte et al. (1994) have approached the problem of defining population response profiles by scanning ellipsoidal surfaces across the receptive fields of peripheral fibers; for moving stimuli the spatial properties of the stimulus may be represented by both spatial and temporal features of the population responses (Johnson and Hsiao, 1992).

In reality the population responses are complicated by two factors. First, the different sensitivities of different SAIs will "distort" the profiles to some degree. Nevertheless, this "distorted" profile will change shape with changes in the shape of the stimulus and will be scaled with changes in the contact force. Whether the brain works directly with these profiles or removes the "distortion" somehow using stored knowledge of the sensitivities of individual fibers, all the psychophysical observations so far could be explained. Sccond, the profiles are sampled by the finite innervation density. The estimated SAI density of about $1 \mathrm{~mm}^{-2}$ (Johansson and Vallbo, 1979; Darian-Smith and Kenins, 1980) is sufficient to preserve the required detail in the profiles. Although most of the RAs tested responded to our stimuli, they responded weakly with no consistent changes with changes in the curvature of the stimulus. Thus, for our stimuli, the RA population is unlikely to contribute much to the human ability to determine shape or contact force during passive touch.
However, since they are active and do have a high innervation density of 1-2 $\mathrm{mm}^{-2}$ (Johansson and Vallbo, 1979; DarianSmith and Kenins, 1980), the RAs may provide cues that are important in certain specific tasks even with stimuli such as ours. In cases where the fingers move over the object or where the object is scanned over the skin as in the studies of LaMotte and Srinivasan (1987b), the RAs probably play an important role in improving shape discrimination. Since $80 \%$ of the PCs did not respond and since they have a low innervation density (Kumamoto et al., 1993), they are probably not significant for our stimuli.

\section{Validity of results}

All SAIs in our sample had similar curvature-response functions to stimuli at the center of the receptive field and similar spatial response profiles; they differed only by a scaling constant equivalent to the sensitivity of the fiber. The consistency of the data is remarkable considering the potential sources of variability unavoidable in a study like this. First, the data came from 14 different monkeys of two different species with a large range of finger sizes, finger curvatures, and skin compliance. Second, even though we only studicd afferents if thcir receptive ficlds were on the central portion of the fingerpad, most were not exactly at the center and the pads are relatively flat over only a small central subregion. Thus, as the stimulus is moved farther from the center of the receptive field, the skin starts curving away from the stimulus, and the degree will vary for different fibers and for different directions of movement away from the center. Also, at the center of the receptive field the contact force is normal to the skin but becomes progressively less normal as the stimulus moves away from the receptive field center. This deviation is greater for receptive fields located farther away from the center of the fingerpad and is greater for smaller and more curved fingers. Also, some receptive fields were located closer to the interphalangeal crease than others or closer to the distal curvature. Our principal aim in this study was to model the responses from the population of digital nerve fibers innervating the fingerpad of the human. Here the skin is much flatter and curvature is more uniform in all directions. The fact that our monkey data are so consistent strengthens the arguments for accepting it as a first approximation to the situation in the human fingerpad. The effect of the changing curvature of the finger itself is a complex issue (Pubols, 1987) that will have to be taken into account in the next generation of more precise models. One reflection of the finger curvature is seen in the constants $b$ and $c$ in our profile data. For a flat finger $b$ and $c$ would always be equal. In our data they are not and their ratio changes with the curvature of the stimulus. This is consistent with the fact that monkey fingers are quite curved and that the curvatures along the long axis of the finger and in a direction at right angles to this are different.

\section{References}

Burgess PR, Wei JY, Clark FJ, Simon J (1982) Signaling of kinesthetic information by peripheral sensory receptors. Annu Rev Neurosci 5:171-187.

Cohen RH, Vierck CJ Jr (1993a) Population estimates for responses of cutaneous mechanoreceptors to a vertically indenting probe on the glabrous skin of monkeys. Exp Brain Res 94:105-1 19.

Cohen RH, Vierck CJ Jr (1993b) Relationships between touch sensations and estimated population responses of peripheral afferent mechanoreceptors. Exp Brain Res 94:120-130.

Darian-Smith I, Kenins P (1980) Innervation density of mechanore- 
ceptive fibers supplying glabrous skin of the monkey's index finger. J Physiol (Lond) 309:147-155.

DeVries SC, Kappers AML, Koenderink JJ (1993) Shape from stereo: a systematic approach using quadratic surfaces. Percept Psychophys 53:71-80.

Edin BB, Abbs JH (1991) Finger movement responses of cutaneous mechanoreceptors in the dorsal skin of the human hand. J Neurophysiol 65:657-670.

Goodwin AW, Morley JW (1987) Sinusoidal movement of a grating across the monkey's fingerpad: representation of grating and movement features in afferent fiber responses. J Neurosci 7:2168-2180.

Goodwin AW, Pierce ME (1981) Population of quickly adapting mechanoreceptive afferents innervating monkey glabrous skin: representation of two vibrating probes. J Neurophysiol 45:243-253.

Goodwin AW, Wheat HE (1992a) Human tactile discrimination of curvature when contact area with the skin remains constant. Exp Brain Res 88:447-450.

Gondwin AW, Wheat HE (1992b) Magnitude estimation of force when objects with different shapes are applied passively to the fingerpad. Somatosens Mot Res 9:339-344.

Goodwin AW, John KT, Marceglia AH (1991) Tactile discrimination of curvature by humans using only cutaneous information from the fingerpads. Exp Brain Res 86:663-672.

Johansson RS, Vallbo AB (1979) Tactile sensibility in the human hand: relative and absolute densities of four types of mechanoreceptive units in glabrous skin. J Physiol (Lond) 286:283-300.

Johansson RS, Landstrom U, Lundstrom R (1982) Sensitivity to edges of mechanoreceptive afferent units innervating the glabrous skin of the human hand. Brain Res 244:27-32.

Johnson KO (1974) Reconstruction of population response to a vibratory stimulus in quickly adapting mechanoreceptive afferent fiber population innervating glabrous skin of the monkey. J Neurophysiol $37: 48-72$.

Johnson KO, Hsiao SS (1992) Neural mechanisms of tactual form and texture perception. Annu Rev Neurosci 15:227-250.

Kumamoto K, Senuma H, Ebara S, Matsuura T (1993) Distribution of pacinian corpuscles in the hand of the monkey, Macaca fuscata. J Anat 183:149-154.

LaMotte RH, Srinivasan MA (1987a) Tactile discrimination of shape: responses of slowly adapting mechanoreceptive afferents to a step stroked across the monkey fingerpad. J Neurosci 7:1655-1671.

LaMotte RH, Srinivasan MA (1987b) Tactile discrimination of shape: responses of rapidly adapting mechanoreceptive afferents to a step stroked across the monkey fingerpad. J Neurosci 7:1672-1681.

LaMotte RH, Srinivasan MA (1993) Responses of cutaneous mech- anoreceptors to the shape of objects applied to the primate fingerpad. Acta Psychol (Amst) 84:41-51.

LaMotte RH, Srinivasan MA, Lu C, Klusch-Petersen A (1994) Cutaneous neural codes for shape. Can J Physiol Pharmacol, in press.

Lederman SJ, Klatzky RL (1987) Hand movements: a window into haptic object recognition. Cognit Psychol 19:342-368.

Marshall S (1989) Review of shape coding techniqucs. Image Vision Comput 7:281-294.

Phillips JR, Johnson KO (1981a) Tactile spatial resolution. II. Neural representation of bars, edges, and gratings in monkey primary afferents. J Neurophysiol 46:1192-1203.

Phillips JR, Johnson KO (1981b) Tactile spatial resolution. III. A continuum mechanics model of skin predicting mechanoreceptor responses to bars, edges, and gratings. J Neurophysiol 46:1204-1225.

Pubols BH (1982) Factors affecting cutaneous mechanoreceptor response. II. Changes in mechanical properties of skin with repeated stimulation. J Neurophysiol 47:530-542.

Pubols RH (1987) Effects of mechanical stimulus spread across glabrous skin of raccoon and squirrel monkey hand on tactile primary afferent fiber discharge. Somatosens Res 4:272-308.

Richards W, Dawson B, Whittington D (1986) Encoding contour shape by curvature extrema. J Opt Soc Am 3:1483-1491.

Roland PE, Mortensen E (1987) Somatosensory detection of microgeometry, macrogeometry and kinesthesia in man. Brain Res Rev 12: $1-42$.

Sathian K, Goodwin AW, John KT, Darian-Smith I (1989) Perceived roughness of a grating: correlation with responses of mechanoreceptive afferents innervating the monkey's fingerpad. J Neurosci 9:12731279.

Srinivasan MA (1989) Surface deflection of primate fingertip under line load. J Biomech 22:343-349.

Srinivasan MA, LaMotte RH (1987) Tactile discrimination of shape: responses of slowly and rapidly adapting mechanoreceptive afferents to a step indented into the monkey fingerpad. J Neurosci 7:16821697.

Talbot WH, Darian-Smith I, Kornhuber HH, Mountcastle VB (1968) The sense of flutter-vibration: comparison of the human capacity with response patterns of mechanoreceptive afferents from the monkey hand. J Neurophysiol 31:301-334.

Vallbo AB, Johansson RS (1984) Properties of cutaneous mechanoreceptors in the human hand related to touch sensation. Hum Neurobiol 3:3-14.

Vierck CJ (1979) Comparison of punctate, edge and surface stimulation of peripheral, slowly adapting, cutaneous afferent units of cat. Brain Res 175:155-159. 